\title{
Stereotaxic Placement of Cannulae in Cerebral Ventricles of the Pig
}

\author{
J. STEVEN POCETA, MICHAEL N. HAMLIN, DAVID W. HAACK, AND DAVID F, \\ BOHR \\ Departments of Physiology and Anatomy, The University of Michigan Medical School, \\ Ann Arbor
}

\begin{abstract}
We have developed and employed a stereotaxic coordinate system for the pig brain based on external skull landmarks. Sagittal, coronal, and horizontal planes were defined. Based on histological maps and ventricular casts, the coordinates for locating the lateral ventricles were described. Guide tubes leading to the lateral ventricles have been chronically implanted. This access route to the ventricular system has been used for stimulation of the dipsogenic response with angiotensin and for withdrawal of cerebrospinal fluid. Solved and unsolved problems arising with these procedures have been defined.
\end{abstract}

Knowledge of the positions of the cerebral ventricles facilitates study of the role of the adjacent nuclei and of cerebrospinal fluid (CSF) in physiological, pathological, and behavioral processes. Using sterotaxic information available in the literature, electrodes and cannulae can be positioned accurately in or near the ventricles of the cat, monkey, dog, and other species (Gergen and MacLean, '62; Horsley and Clarke, '08; DeGroot, '59; Lim et al., '60; Knigge and Joseph, '63). However, detailed sterotaxic information for the pig does not exist. Fitzgerald ('61) has demonstrated the shape and size of the ventricular system of the pig, but the latex models he produced were without reference to external skull markings. Similarly, Yoshikawa's atlas ('68) of the pig brain does not provide stereotaxic reference. Dellmann and McClure ('65) studied the pig skull and determined preliminary stereotaxic planes, although they did not describe an implementation of their system.

Studies in our laboratory (Miller et al., '79) have established that the pig serves as a very satisfactory model for the study of mineralocorticoid hypertension. Brody et al., ('78) have demonstrated the importance of the region of the anteroventral third ventricle in the development of this type of hypertension. In order to evaluate the role of the central nervous system in this model of hypertension in the pig, we have developed an access to the lateral cerebral ventricles to be described in this study.

We have established and employed a stereotaxic coordinate system for the pig brain based on external skull landmarks. The stereotaxic system to be described is easily reproduced, and is suitable for placement of cannulae or electrodes into and about the cerebral ventricular system of the young pig. We have also attempted to maintain chronically implanted cannulae in their brains. It is intended that this work serve as a reference for future investigation and mapping of specific brain structures in the pig.

\section{MATERIALS AND METHODS}

Male Yorkshire pigs (sus scrofa domesticus), aged 4-5 months and weighing $38-45 \mathrm{~kg}$, were purchased from a local farm. These animals gained weight at the rate of $0.5 \mathrm{~kg}$ per day. At the time of sacrifice, pigs were anesthetized with sodium pentobarbitol ( $50 \mathrm{mg} / \mathrm{kg}$ body wt.). In some pigs, approximately $4 \mathrm{ml}$ of CSF was withdrawn from the cisterna magna prior to fixation of the brain. Both common carotid arteries were exposed and cannulated. The head was then perfused with $5 \%$ formaldehyde in saline via the carotid cannulae at a pressure of $120 \mathrm{~cm} \mathrm{H}_{2} \mathrm{O}$. The external jugular veins were severed to allow for drainage of fixative and blood. At least 2 liters of fixative were perfused. The heads were removed, and 24 hours later the hardened brains were exposed by careful removal of the calvaria.

Received November 6, 1980; accepted December 30, 1980). 


\section{Stereotaxic methods}

The stereotaxic device used to align and secure the pigs' heads was an adaptation of a David Kopf (Tujunga, California) dog stereotaxic device (model 1530). The stereotaxic bars of the original frame were supported by 1.27$\mathrm{cm}$ aluminum bars, and were separated to allow for the greater width of the pig's head (Fig. 1). The device supported the head at the bony ear canals by means of ear bars, and at the mouth by an adjustable crossbar. The external ear canals of the pig enter the skull at an angle $40^{\circ}$ ventral to the horizontal plane, as defined in Figure 2, and $19^{\circ}$ rostral to the coronal plane through the external auditory meati. Therefore the ear bars, constructed of $6.5 \mathrm{~mm}$ square steel rod, were tapered and angled so as to extend into the bony ear canal and lodge tightly without damaging the canal or the tympanic membrane.

To ascertain stereotaxic planes of reference, a head was placed in the apparatus so that the ear bars held the head securely. The mouth bar was then adjusted to place the external acoustic meati and the ventral lacrimal foraminae in the same horizontal plane, as is done for most animal stereotaxy (Horsley and Clarke, '08). This is the zero horizontal plane (Fig. 2). In this position, the surface of the skull at the bregma forms a $15^{\circ}$ angle with the horizontal plane. The zero sagittal plane is defined by the sagittal suture of the skull. The zero coronal plane passes through the center of the external acoustic meati, and is perpendicular to the zero horizontal plane. This definition of planes is the same as that used by Dellman and McClure ('65).

\section{Graphical reconstruction of ventricles}

Three of the heads were placed in the sterotaxic device, and eleven 20-gauge marker needles were lowered into each brain perpendicular to the horizontal stereotaxic plane at specific stereotaxic coordinates. Needles were also passed through the brain perpendicular to these needles and to the coronal plane in an anteroposterior direction. These needles provided reference markings that could later be related to stereotaxic coordinates. The brains were sliced in the coronal plane into 4-mm slabs. The slabs were dehydrated, embedded in paraffin, and sectioned at a thickness of 10 $\mu \mathrm{m}$. Every 25 th section was stained with Harris's hematoxylin and alcoholic eosin.
Two heads were used to make latex casts of the ventricular system. An 18-gauge needle was placed in each of the lateral ventricles at the level of the interventricular foramen. Approximately $10 \mathrm{ml}$ of latex (Vinyl Acetate Solution, Wards Natural Science Establishment) was injected and observed to flow out of the cisterna magna. The heads were then dissolved in concentrated hydrochloric acid leaving the latex cast of the ventricular system.

The histological sections and the latex casts provided material for reconstruction of the ventricles. First each histological section was projected using a microprojector, and the ventricles were traced on graph paper. Then measurements were made from the reference marks (made by the needles) to specific points on the lateral and third ventricles. Lateral and dorsal views of the ventricular system were generated by transferring key points from the ventricular system at each coronal level to other graphs. Coronal sections were processed in register by reference to the anteroposterior needle tracts. Shrinkage of tissue due to the various procedures was calculated by noting changes in distance between reference needle markings. It was found to be approximately $12 \%$. Shrinkage was taken into account in the graphical reconstruction. Variations between the three reconstructed ventricular systems were less than $10 \%$, and were averaged into a composite picture.

\section{Chronic studies}

Stereotaxic coordinates were used to implant cannulae into the cerebral ventricles. A system of cannulae with guide tubes, modeled after that of Pappenheimer et al. ('62) was developed. With the animal's head in the stereotaxic device and using the coordinates taken from our reconstructions of the lateral ventrical (Figs. 3 and 4), we lowered a guide tube through a burr hole in the skull and through the cerebral cortex. A slow infusion of artificial cerebrospinal fluid was delivered through the guide tube so that penetration of the ventricle could be signaled by an abrupt fall in pressure in the infused fluid. Guide tubes with stylets were secured to the skull with dental acrylic (Perm Rebase Repair Acrylic, Hygienic Dental Mfg. Co., Akron, Ohio) and screws. To gain access to the cerebrospinal fluid, the stylets were removed and cannulae were inserted into the ventricles. Evidence that the cannulae were in the ventricular space came from perfusate pressure changes at the time of surgical 

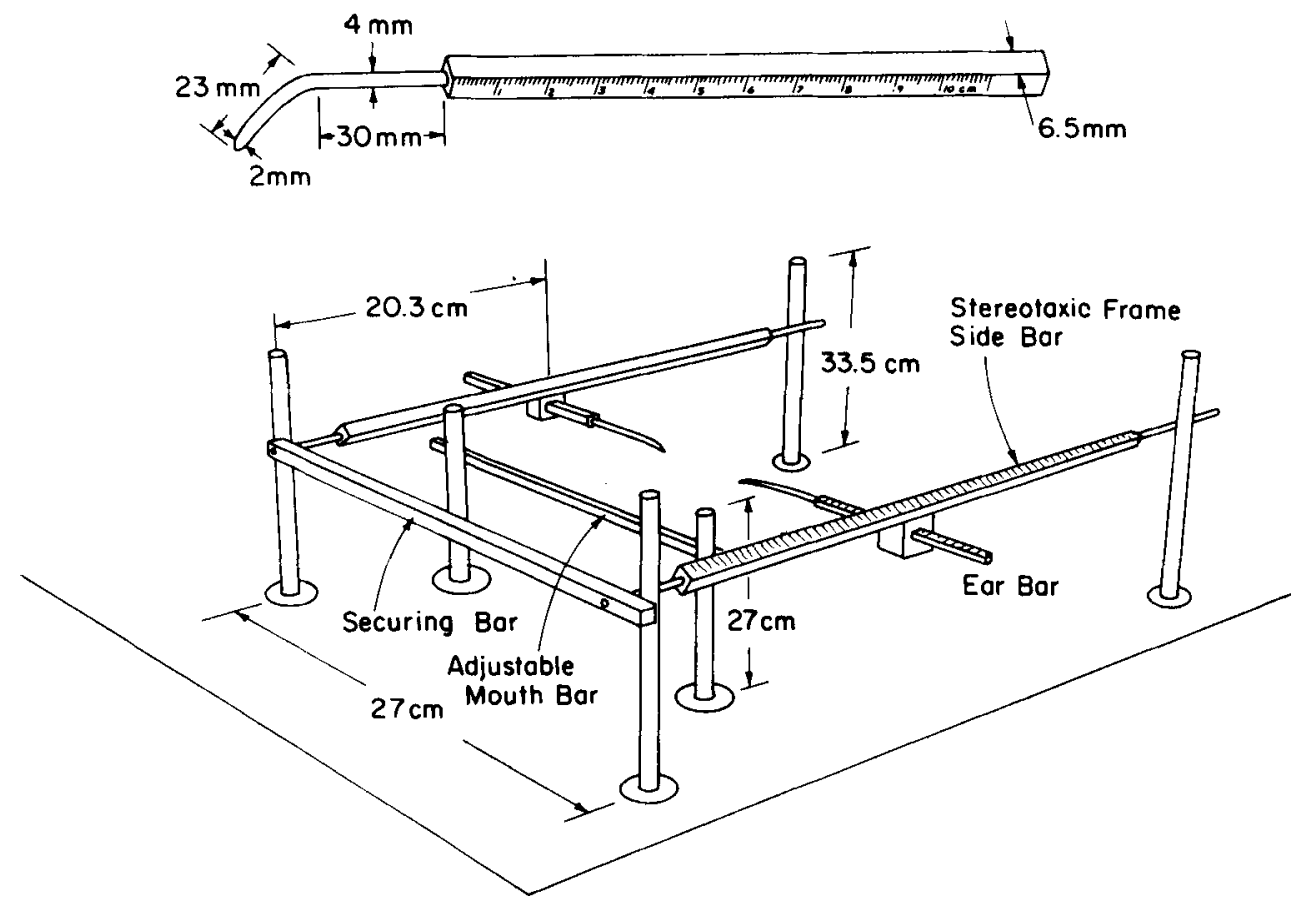

Fig. 1. Stereotaxic apparatus and ear bar. The adjustable mouth piece was placed between the teeth of the pig. The base is of wood, support bars are of aluminum, and frame and ear bars are of steel.

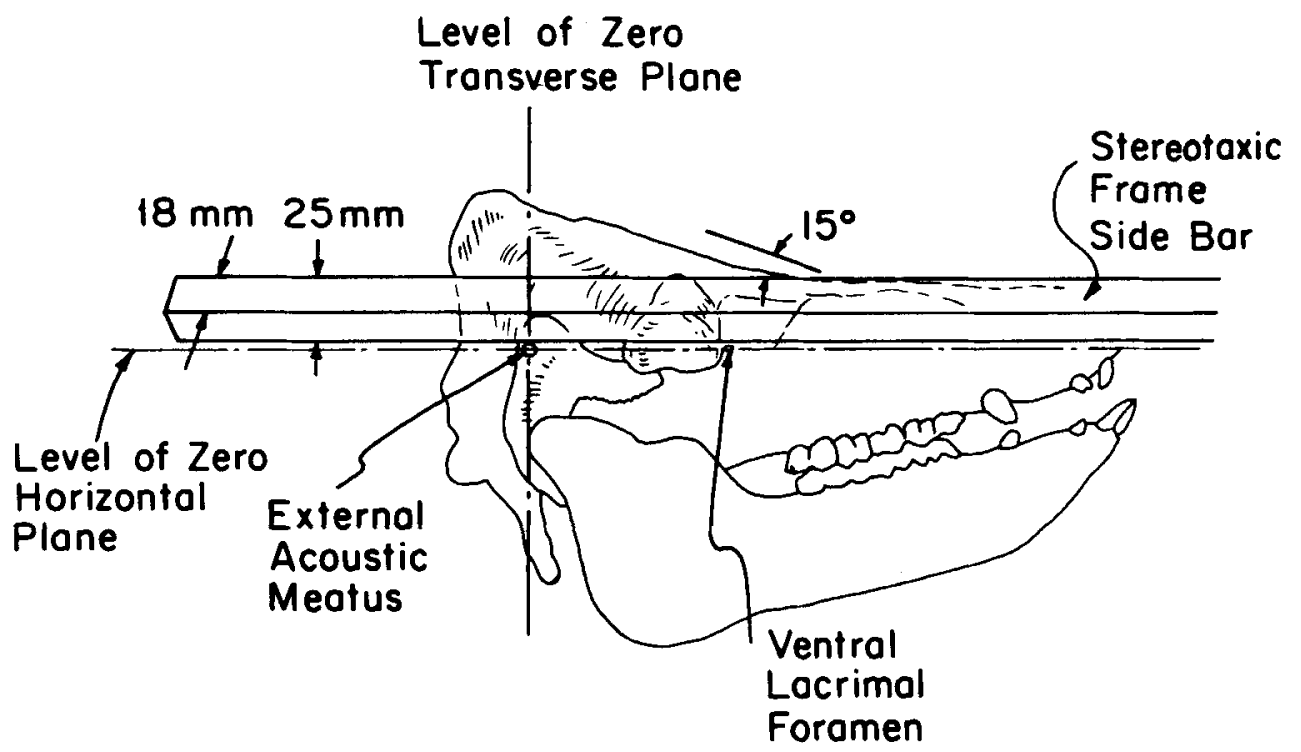

Fig. 2. Proper alignment of pig skull relative to stereotaxic frame. The ventral lacrimal foramen and the external acoustic meatus are in the same horizontal plane. The levels of the horizontal and transverse coordinate planes are drawn in dashed lines. 


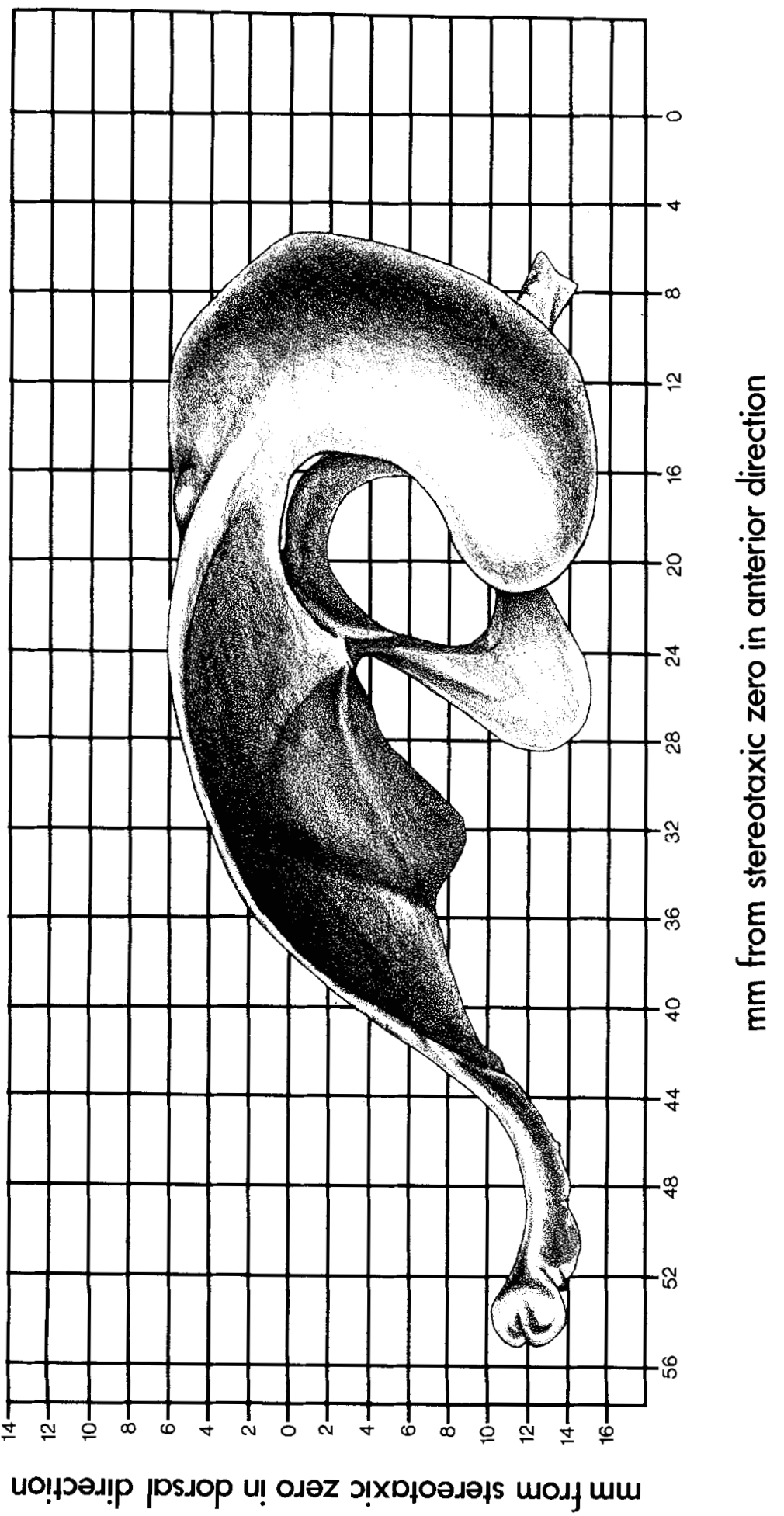


placement (Pappenheimer et al., '62). postmortem examination of the staining from Evans's blue dye injected into the cannulae at sacrifice, and the animals' response to infusions into the cannulae of angiotensin II. Angiotensin II (Ciba, Hypertensin) at a concentration of $10 \mu \mathrm{g} / \mathrm{ml}$ in artificial CSF (Mouw et al., '74) was delivered at a rate of $0.1 \mathrm{ml} / \mathrm{min}$ for $5 \mathrm{~min}$. A Harvard infusion pump located outside the cage was used to deliver the drug. The vehicle alone was infused for 5 min prior to the angiotensin II infusion. Withdrawal of CSF was performed by gravity suction while the animal way lying strapped on its side. Sometimes anesthesia (Surital, $4 \mathrm{mg} / \mathrm{kg}$ body wt.) was used at the time of CSF removal. Five pigs received guide tube implants, and angiotensin II infusion studies were carried out in three.

\section{RESULTS}

The cerebral ventricular system of the brain of the young pig has been mapped by means of histological sections and latex casts. Stereotaxic coordinates for certain structures are presented in Table 1. The extent and location of the ventricular system are presented in Figures 3 and 4 .
The three criteria of proper cannulae placement (see Materials and Methods) indicate that the stereotaxic coordinates are adequate for placement of cannulae into the lateral ventricles. Infusion of angiotensin II into the cannulae resulted in an immediate dipsogenic response in all cases (three of three attempts). When approximately $0.6 \mu \mathrm{g}$ angiotensin II was delivered to the ventricle, the animals went to their water pans and drank over 1 liter. Infusion of the artificial CSF alone did not result in any drinking behavior. Additionally, abrupt falls in perfusate pressure during placement of cannulae, and postmortem dye examination revealed proper cannula placement in all five pigs. CSF removal, however, was successful in only two pigs.

Chronic cerebral implant in the pig is possible, and the surgery was without ill effect on the animal. However, the pig is a difficult animal model to use for chronic experiments, and our guide tubes remained in place for only relatively short periods. The young pig is a rambunctious and strong animal, capable of knocking off the dental acrylic which secures the guide tube. The restless nature of the pig made aseptic handling of the cannulae difficult in the unanesthetized animal.

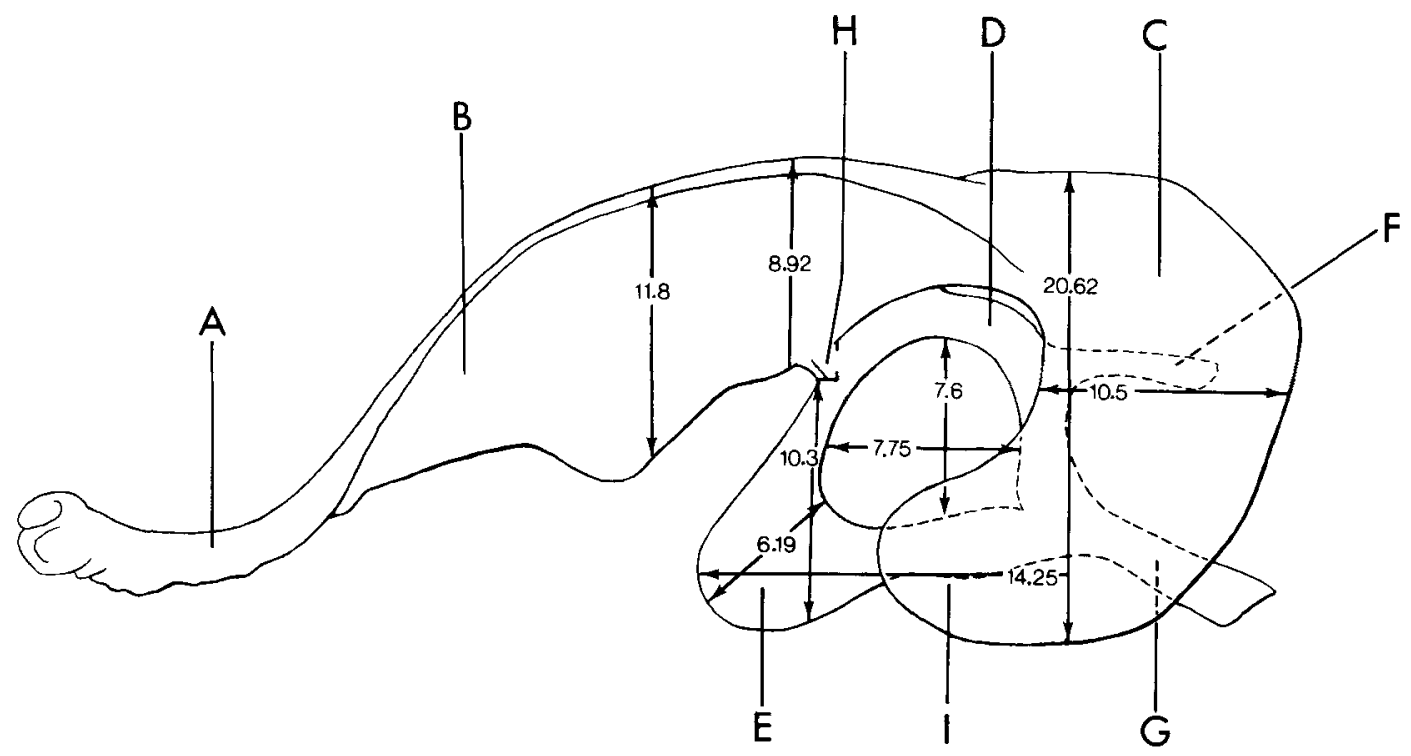

Fig. 3. At left is artist's sketch of ventricles with stereotaxic coordinates - lateral view. Each axis is labeled in millimeters from stereotaxic zero. In the sketch above $A$ is the olfactory stem of lateral ventricle; $B$ is the rostral horn of lateral ventricle; $\mathrm{C}$ is the body of lateral ventricle; $\mathrm{D}$ is the third ventricle; $\mathrm{E}$ is the infundibular region of third ventricle; $\mathrm{F}$ is the suprapineal process of third ventricle; $G$ is the cerebral aqueduct (aqueduct of Sylvius); $H$ is the interventricular foramen; $I$ is the inferior horn of lateral ventricle. Numbers represent distance in millimeters. 


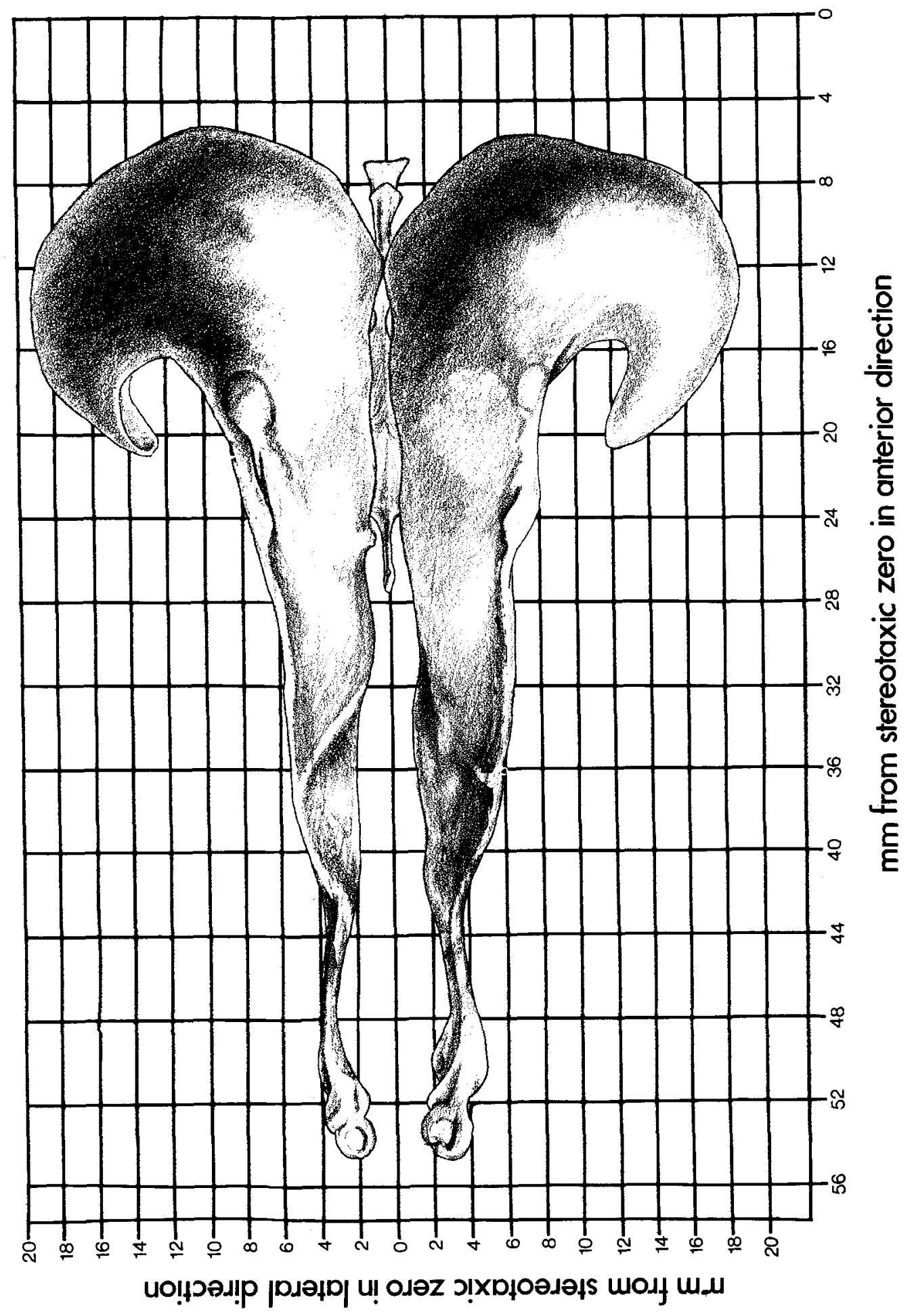




\section{DISCUSSION}

This report describes a modification of a commercially available stereotaxic device and provides a system of stereotaxic coordinates that allows accurate and reproducible placement of cannulae or electrodes into or near the cerebral ventricular system of the pig.
A number of problems that are unique to the pig were encountered in this study. First, the size of the pig head required that the stereotaxic device be widened and reinforced. Second, the morphology of the external auditory meati required the use of curved ear bars. Finally, mapping of the ventricular system may be subject to errors introduced by shrinkage and dis-

TABLE 1. Stereotaxic coordinates

\begin{tabular}{lccc}
\hline \multicolumn{1}{c}{ Structure } & AP & DV & ML \\
\hline $\begin{array}{l}\text { bregma } \\
\begin{array}{l}\text { AV } 3 \text { area } \\
\text { (circumventricular) }\end{array}\end{array}$ & $+13.6 \pm 3.4$ & $+45.8 \pm 4.7$ & 0.0 \\
$\begin{array}{l}\text { Interventricular } \\
\text { foramen }\end{array}$ & $+27.8 \pm 0.8$ & $-14.0 \pm 0.3$ & 0.0 \\
$\begin{array}{l}\text { Target spot for } \\
\text { lateral ventricle } \\
\text { cannula }\end{array}$ & $+23.4 \pm 1.3$ & $-3.0 \pm 0.4$ & 0.0 \\
\hline
\end{tabular}

AP: $m m \pm$ S.D. from stereotaxic zero in anterior direction.

DV: $m m \pm S . D$. from stereotaxic zero in dorsal direction.

ML: $\mathrm{mm} \pm \mathrm{S} . \mathrm{D}$. from stereotaxic zero in lateral direction.

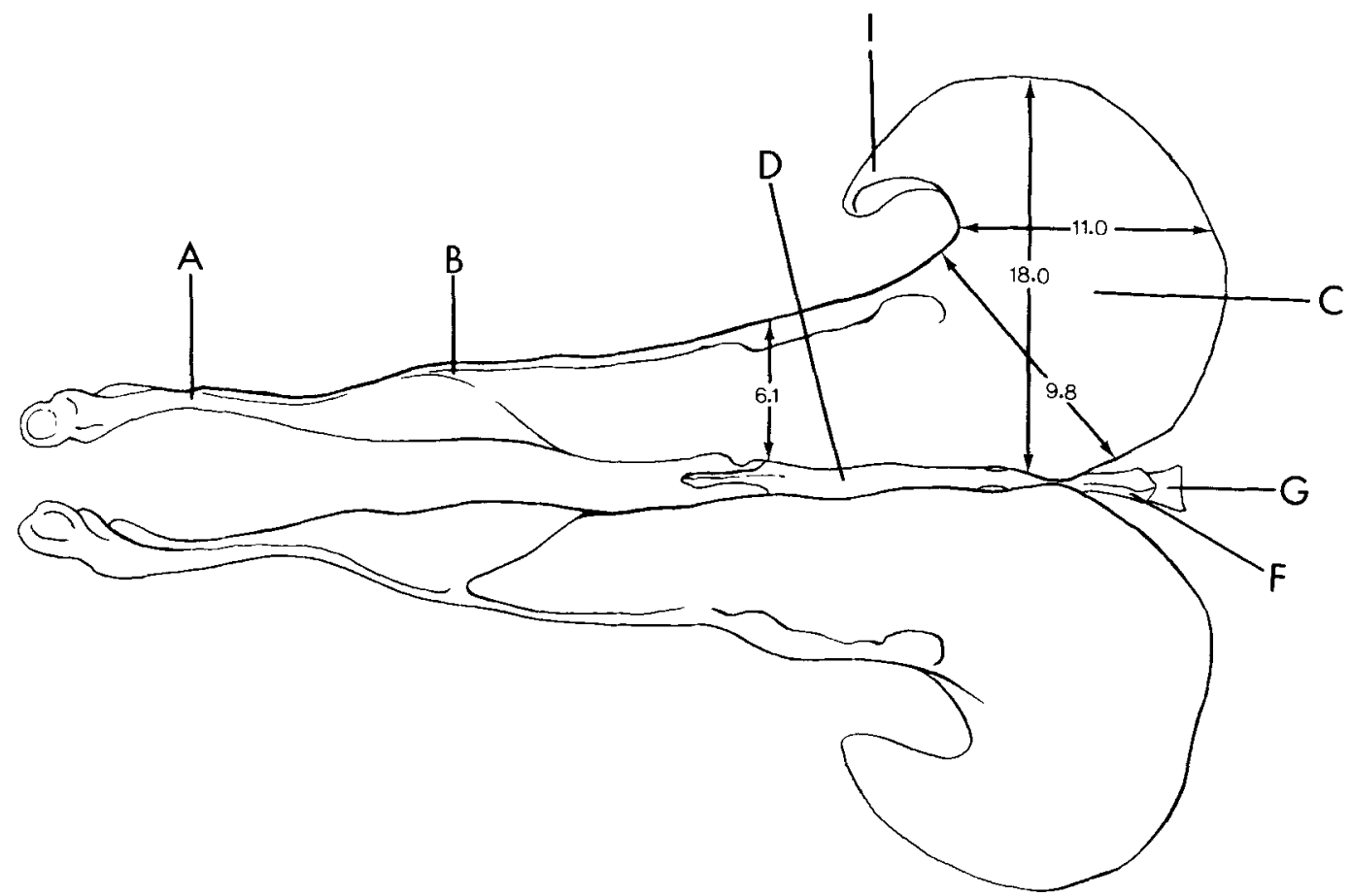

Fig. 4. At left is artist's sketch of ventricles with stereotaxic coordinates-dorsal view. Each axis is labeled in millimeters from stereotaxic zero. In the sketch above, letters designate the same structures as in Figure 3. Numbers represent distance in millimeters. 
tortion of the ventricular casts and histological preparations (MacFarland et al, '69). However, by use of a combination of the systems of ventricular mapping and casting we have compensated for changes in the measurement of the ventricular boundaries.

A second set of problems remains to be resolved. The first of these is the design of an anchoring system or a protective helmet that will prevent the pig from dislodging chronically implanted cannulae. Second, CSF removal was not usually successful, possibly because the ventricle collapses under negative pressure, or because the choroid plexus plugs the cannulae. Finally, the young pig grows rapidly, approximately $0.5 \mathrm{~kg} / \mathrm{day}$, and skull thickness increases proportionally. This presents a problem for chronic studies because the increasing thickness of the skull causes a shift of the skull-mounted cannulae relative to the ventricles.

There is much recent interest in the role of the central nervous system in various responses in hypertension (Brody et al., '78; Buggy et al., '77; Haeusler et al., '72; Hoffman et al., '77). In particular, the nuclei of the region near the anteroventral side of third ventricle (AV3V) have been under study for a possible role in various forms of hypertension. The pig is well suited for studies of the cardiovascular system in hypertension (Miller et al., 79), and we include stereotaxic coordinates for the AV3V region in this report. This study provides a background for future investigation of the central nervous system in the pig.

\section{ACKNOWLEDGEMENTS}

We give special thanks to Drs. Sarah S. Winans, David R. Mouw, and Samuel P. Hicks for their generous guidance in this study. We thank Ms. Martha Lynn Hefner for her artful reconstruction of the ventricles.

These studies were supported by grant No. HL 18575 from the National Institutes of Health.

\section{LITERATURE CITED}

Brody, M.J., G.D. Fink, J. Buggy, J.R. Haywood, F.J. Gordon, and A.K. Johnson (1978) The role of the anteroventral third ventricle (AV3V) region in experimental hypertension. Circ. Res. 43, Suppl. 1:I-2-I-13.

Buggy, J., W.E. Hoffman, and A.K. Johnson (1977) Preoptichypothalamic periventricular ablation alters $\mathrm{ADH}$ release and attenuates pressor responses to intracerebroventricular injections of angiotensin and hyperosmotic solutions. Soc. Neurosci. Abstr. 3:340.

DeGroot, J. (1959) "The rat forebrain in stereotaxic coordinates." N.V. Noord-Hollandsche Vitgeners Maatschappiji, Amsterdam.

Dellman, H.D., and R.C. McClure (1965) Skull measurements for the establishment of a coordinate system for stereotaxic placement in the brain of swine (sus scrofa). In: Swine in Biomedical Research, Proceedings of a Symposium at Pacific Northwest Laboratory, Richland, Washington. Frayn Printing Co., Seattle, pp. 537-542.

Fitzgerald, T.C. (1961) Anatomy of cerebral ventricles of domestic animals. Vet. Med. 56, 1:38-45.

Gergen, J.A., and P. Maclean (1962) A stereotaxic atlas of the squirrel monkey's brain. U.S. Govt. Printing Office, No. QL 937.G43., p. 91.

Haeusler, G., L. Finch, and H. Thoenen (1972) Central adrenergic neurons and the initiation and development of experimental hypertension. Experientia 28:1200-1203.

Hoffman, W.E., J. Buggy, and P.G. Schmid (1977) Effect of central catecholamine depletion on centrally mediated cardiovascular responses. Soc. Neurosci. Abstr. 3:346.

Horsley, V., and P.H. Clarke (1908) The structure and function of the cerebellum explained by a new method. Brain $31: 45-124$.

Knigge, K., and S.A. Joseph (1963) "The stereotaxic atlas of the brain of the golden hamster." Directorate of Medical Research, Chemical Research and Development Laboratories, U.S. Army, Edgewood Arsenal, Maryland.

Lim, R.K.S., C. Liu, and R.L. Moffitt (1960) "A stereotaxic atlas of the dog's brain." Charles G. Thomas, Springfield, Illinois.

McFarland, W., P.J. Morange, and M.S. Jacobs (1969) Ventricular system of the brain of the dolphin, Tursigus trun catus, with comparative observation and brain relation to brain specializations. J. Comp. Neurol. 135, 3:275-367.

Miller, A.N., D.F. Bohr, A.M. Schork, and J.M. Terris (1979) Hemodynamic responses to DOCA in young pigs. Hypertension 1:591-547.

Mouw, D.R., S.F. Abraham, and J.S. McKenzie (1974) Use of ventriculo-cisternal perfusion in conscious sheep. Lab Anim. Sci. 24, 3:505-509.

Pappenheimer, J.R., S.R. Heisey, E.F. Jordan, et al. (1962) Perfusion of the cerebral ventricular system in unanaesthetized goats. Am. J. Physiol. 203:763-774.

Yoshikawa, T. (1968) "Atlas of the brains of domestic animals." University of Tokyo Press, Tokyo. 